\title{
Factors influencing cloud computing adoption in higher education institution
}

\author{
Wan Abdul Rahim Wan Mohd Isa, Ahmad Iqbal Hakim Suhaimi, Nurulhuda Noordin, \\ Afdallyna Fathiyah Harun, Juhaida Ismail, Rosshidayu Awang Teh \\ Faculty of Computer and Mathematical Sciences, Universiti Teknologi MARA, Malaysia
}

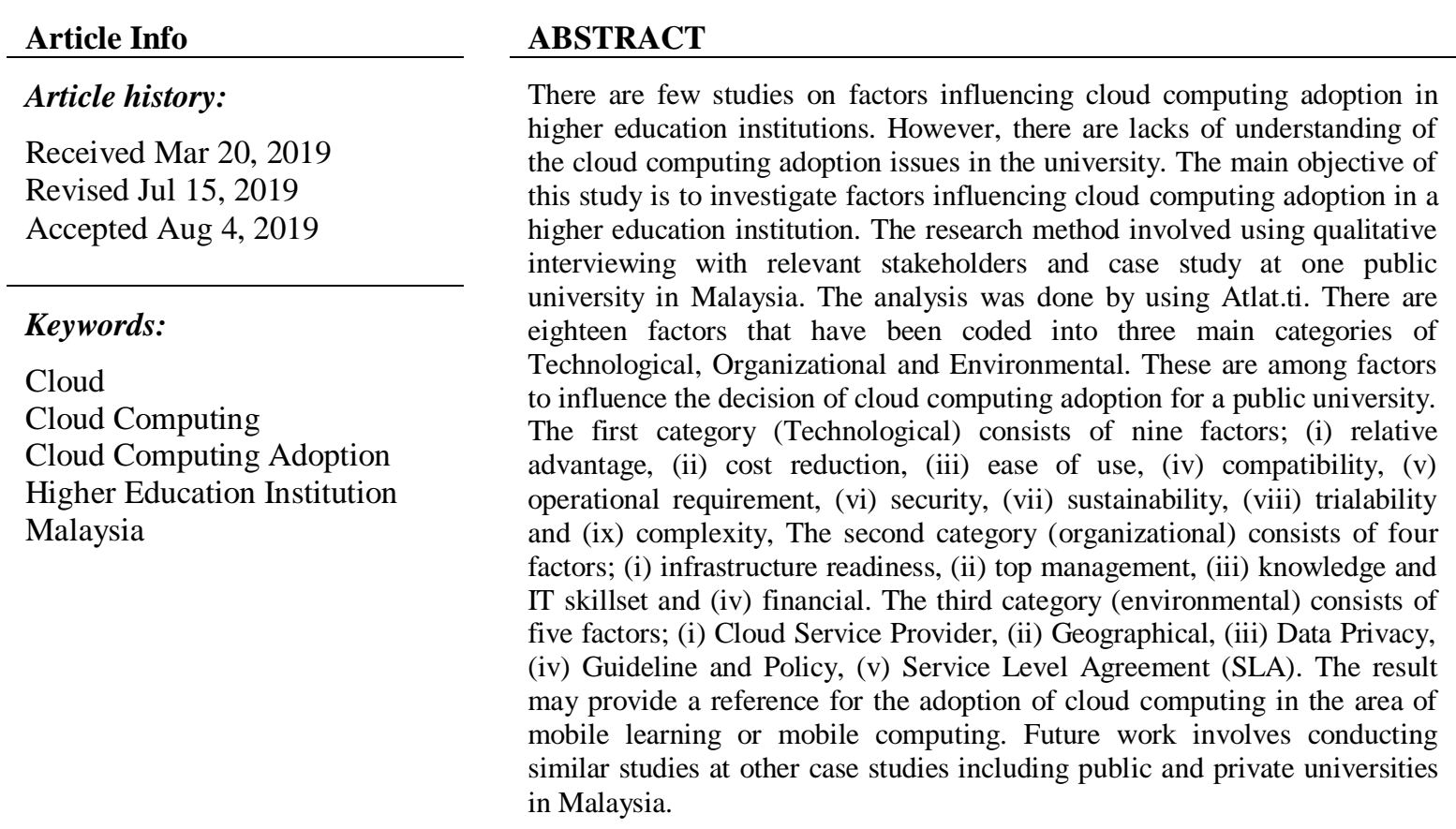

Copyright $(0) 2020$ Institute of Advanced Engineering and Science. All rights reserved.

Corresponding Author:

Wan Abdul Rahim Wan Mohd Isa,

Faculty of Computer and Mathematical Sciences,

Universiti Teknologi MARA,

40450 Shah Alam, Selangor, Malaysia.

Email: wrahim2@tmsk.uitm.edu.my

\section{INTRODUCTION}

Cloud computing is an innovative technology that introduces the idea of information sharing, consolidation of hardware and software, as well as to standardize infrastructure resources in a centralized facility [1]. This concept offers many advantages for economic, technology and society. Cloud computing has been widely used in industries as well as government sectors. Information System success model indicating that IT innovation offers many benefits to cloud computing adoption and offers many benefits to an individual or even an organization. The adoption of the technology usually associated with a decision to adopt or not to adopt. Researchers who conducted studies on cloud computing adoption has identified several important factors that influence the decision-maker towards cloud computing adoption such as top management, trialability, cost reduction, firm size, cloud service provider support, infrastructure readiness, competitive pressure, security and others. Innovation factors that have the most consistently significant to innovation adoption are compatibility, relative advantage and complexity [2]. The compatibility is perceived 
workable with the existing infrastructure and meet the adopter's needs [3]. The relative advantage is viewed as an advantage for an organization to perform the task in different ways and the complexity is perceived as difficult to understand and use [3].

The advantages of cloud computing are reducing cost, scalability, flexibility, mobility, speed, usefulness, quality, convenience, performance and shared resources [4-6]. Cloud computing has many important characteristics to explain the usage of it. There are four characteristics describes by NIST [7] which are on-demand self-service, broad network access, resource pooling, rapid elasticity and measured services. On-demand self-services refers to capability provisioning infrastructure by the customer without the intervention of service provider. Thus, they pay the usage based on their consumption [8]. Mean while, broad network access defines compatibility to heterogeneous of the platform by using a standard mechanism. Resource pooling describes the role of a service provider that handling a multi-tenant model for cloud resources in servicing the customer requirement. Rapid elasticity means unlimited resources during provisioning leads to any quantity at any time. Lastly, measured services is a metering capability that providing transparency for customer and service provider. Cloud service categorized into three models which are Software as a Service (SaaS) that provides the ability to run software on the cloud and customer can access via the Internet to work on it. Platform as a Service (PaaS) meant for expanding the ideas and cooperate when developing programs and software by the system developer. At this layer, the developer may focus on the innovation rather than aware of infrastructure problems. Infrastructure as a Service (IaaS) considered as a foundation level for the cloud computing platforms that managed by customers [7, 9, 10].

There are four cloud computing deployment models for cloud architecture solutions according to NIST and ISO/IEC which are private, public, hybrid and community. Private describes the organization's data centre or application operated on-premises. It is considered more secure than the public cloud. Whereas public cloud meant for data centre or application operated outside the organizations' premises. Hybrid can be described as a mixture of two different deployment models. The community model is a public cloud that suitable for certain targeted groups of organizations that share the same objectives [9, 10]. As one of emerging technology, cloud computing has rapidly spread in academic institutions. Therefore, adopting cloud computing technology as an educational service enabler is very significant to a dynamic learning style. The technology offers democracy in learning delivery of IT services as well as availability at anytime, anywhere and from any devices [11]. It also offers efficient collaboration and information sharing platform, obtains fairness in educational services and simplifies the learning process, eliminates the spatial and temporal boundaries [12].

Able to reduce capital expenditure by minimizing cost for the education environment including provides free maintenance such as the usage of email by Google. The SaaS application is significance as daily communication medium whether in the academic or non-academic area such as email, collaboration services, and storage services [13]. Besides that, it offers an independent learning approach, for example, using online meeting spaces, video conferencing and video chat are allowing the student to meet and attend classes online [14]. Cloud location having the geographical advantage where it is located a thousand miles away, enables customer data safe and recovery at anytime and anywhere. Thus meet the requirement for disaster recovery plan [11]. It also can support a wide range of applications running on different hardware devices like smartphones, tablets, and PDAs. The new generation of students empowered to involve in the learning process and play an active role in sharing and collaborative learning and not only retrieve the information [15].

There are few studies of cloud computing adoptions in the area of (i) Social Networking [16], (ii) Telecommunication [17], and (iii) higher education institutions [18]. However, there are lacks of understanding of the cloud computing adoption issues in the university. There are many other factors that also explain the adoption rate in an organization. The existence of theory, framework, and model are proven to help in understanding the factor and the process of adoption of technology innovations [19]. The Technological-Organizational- Environmental (TOE) framework by Tornatzky and Fleischer, the Diffusion of Innovation (DoI) theory by Rogers and the Theory Acceptance Model (TAM) are frequently used among researchers to explain technology innovation in the ICT acceptance and adoption in the organization [20]. In summary, cloud computing perceived many benefits to an organization. A decision to adopt the technology would be influenced by many factors, opportunities, advantages offered as well as the challenges. The main objective of this study is to investigate factors influencing cloud computing adoption in a higher education institution. This theoretical basis of this study derived from the TOE framework and Diffusion of Innovation theory. 


\section{RESEARCH METHOD}

Qualitative research is the research method used in this study. Qualitative research is important when the phenomenon needs to be understood [21]. This research method allows to examine people's experience in detail through certain research methods like the in-depth interview, focus group discussion, observation, content analysis, visual methods, and life histories or biographies [22]. In this study, a case study research approach is employed by applying qualitative interviews towards cloud computing decisionmakers in a public university in Malaysia. The qualitative interview is usually intended to refer to in-depth, semi-structured or loosely structured forms of interviewing [23].

A case study protocol was used throughout the data collection process. The protocol is important to guide for reliable data collection [24]. Semi-structured interviews with ten IT experts and other IT professionals from a public university in Malaysia were conducted from March 2017 until September 2017. The key informants were involved in the cloud computing adoption decision process in the university. The semi-structured interview becomes the primary method for the data collection method. The semi-structured is where early questions have been set and it can be modified, omitted or added during the interview session [25]. During the interview process interaction face to face with the informants is important to clarify and ensure understanding of the interview statements [26]. The questionnaires were constructed hugely as openended questions, where it has been verified by the academic expert. The interview was recorded using the voice recorder as an instrument. As a backup, notes also taken during the interview [21].

\section{RESULTS}

The analysis was done by using Atlat.ti. Figure 1 illustrates the factors influencing cloud computing adoption in higher education institution. There were eighteen factors that have been coded into three main categories which were Technological, Organizational and Environmental. There are eighteen factors that have been coded into three main categories of Technological, Organizational and Environmental. The technological category describes the main features of the technology itself that can influence the adoption process in the organization. The factors reflect technology use [1]. The organizational category refers to factors that involve organizational involvement or process. Then, the environmental category refers to the external factors that can influence the adoption of technologies [27].

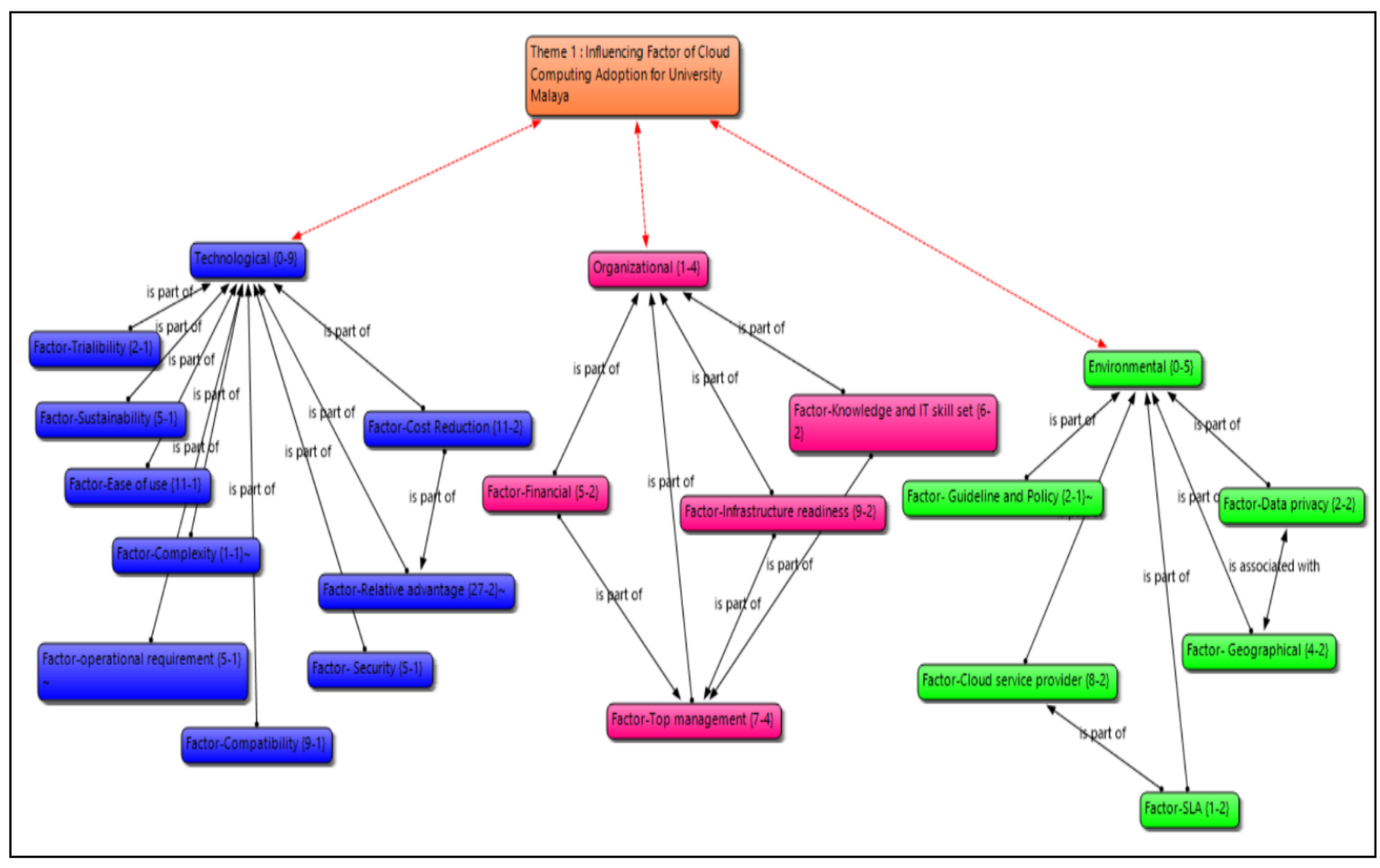

Figure 1. Factors Influencing Cloud Computing Adoption in Higher Education Institution 


\subsection{Technological Category}

The technological category describes the main features of the technology itself that can influence the adoption process in the organization. The factors reflect technology use [1]. The first category (Technological) consists of nine factors; (i) relative advantage, (ii) cost reduction, (iii) ease of use, (iv) compatibility, (v) operational requirement, (vi) security, (vii) sustainability, (viii) trialability and (ix) complexity. The following provides the description.

\subsubsection{Coding Unit 1: Relative Advantage}

The relative advantage becomes the highest influence factor among the university's decision-makers with frequent of 27 quotations from eight key informants. This factor was seen in terms of accessibility, availability, speed and performance, fast server provisioning, flexibility and scalability. For example, fast server provisioning became the main factor to drive the university consolidated one hundred physical servers to three ESX hosts. The university also hosted the main website in the public cloud for fast website development. The speed to provision a new server is important for the website development team to fulfil user needs or meet the dateline. Hence, this factor looks significant in ensuring scalability for the infrastructure, high availability for the customers, access at any time and from anywhere.

\subsubsection{Coding Unit 2: Cost Reduction}

The cost reduction factor also plays a significant role in influencing cloud computing adoption in this organization. The cost reduction is perceived to be part of the relative advantage that can give economicbenefit [26]. This factor also becomes a major driver for the rapid adoption of cloud computing [28] and contribute to the sustainability of organization operating expenditure [29]. The cost reduction was highlighted by six key informants with eleven quotations. The Acting IT Director of the university emphasized on the cost-benefit and the organization must perform a cost-benefit analysis before deciding to adopt the technology.

\subsubsection{Coding Unit 3: Ease of Use}

The ease of use can be explained by an encouraging element toward innovation due to the simplicity of using the technology [30]. Cloud computing found less effort needed and optimize the work time [31]. Most of the key informants consider cloud computing is easy to be used. Whether from perspective Software as a Service (SaaS) or Infrastructure as a Service (IaaS) model. There were eleven quotations form eight key informants expressed about the ease of use when dealing with cloud computing technology.

\subsubsection{Coding Unit 4: Compatibility}

The compatibility is very important to ensure the application works smoothly whether on the same cloud platform, inter-cloud or hybrid deployment. When moving to a cloud environment, the surrounding issue like network must be aware by adopters [6]. There were nine quotations from six key informants. There was one compatibility issue when a web server located at the public cloud platform whereas the database was located in the local university's data centre. The issue led to slowness when browsing the university's website. Thus, by moving the database to the same cloud environment was solved. There could be many reasons when relating to the issue such as network bandwidth or network routing. This shows that resource readiness is very important in cloud adoption.

\subsubsection{Coding Unit 5: Operational Requirement}

The operational requirement is important to achieve the objectives. To move to a new operational environment, require firm justification. Thus, the operational requirement becomes an important element that translated into needs. The university has the requirement to fulfil the nature of the system that requires to develop and hosted in the cloud, the readiness of the cloud platform with tools and template aids in fast server provision, and due to technical challenges.

\subsubsection{Coding Unit 6: Security}

The security is an important factor that can represent trust toward cloud computing services [32]. Research indicates that a private cloud is known to be more secure than the public cloud. Thus, the cloud service provider requires a cloud security standard in ensuring the secure platform that can be perceived secure and safe by the customer [33] There are concerns about the security of cloud computing services among the key informants. They are also aware of the mechanisms to ensure security compliance at the highest levels. The security-compliance will increase the customer's confidence level towards cloud computing adoption. There were five quotations with four key informants raised about this element. 


\subsubsection{Coding Unit 7: Sustainability}

The sustainability means able to maintain technology for a longer time. Choosing the right cloud computing business model is important [34]. The essential element to sustain the technology is in terms of cost. Therefore, sustainability has a strong linkage towards budget allocation of cloud computing in the university. According to the Acting IT Director, the organization may not have considered if the technology is not able to be sustained.

\subsubsection{Coding Unit 8: Trialability}

The trialability is the capability of the technology to be try-out in certain scopes [3]. Based on the theory of diffusion of innovation, there will be adoption rate increment when the adopters able to test the technology [26]. The importance of the factor has been stressed by the Acting Director of the IT Centre during gathering information about cloud computing. The factor supports understanding of the technology before a decision to adopt. This approach works very well especially on Infrastructure as a Service model, when they want to adopt any technology for the organization. However, SaaS adoption like Google's email and Microsoft Office, have not experimented with it as an organizational level because of the similarity with the technology as individual usage.

\subsubsection{Coding Unit 9: Complexity}

The complexity can be linkage with the negative perspective of the adoption of technology. Most practitioners perceived cloud computing as complicated [26]. The complexity hardly influences this organization in the decision to adopt cloud computing. Overall informants' feedbacks showed the easiness of the use of technology. The university has implemented Software as a Service (SaaS) model, which offers a friendly interface without the requirement of handling the technical configuration. Whereas Infrastructure as a Service (IaaS), require them to have understanding and skill on the technical configuration. However, with the experience of managing private cloud VMWare the IT staff able to learn new skills in the public cloud environment. Nevertheless, the complexity factor has been concerned by the Data Centre team to decide which public cloud in the market suit the environment. According to the Project Manager from the Data Centre Management Division, the Microsoft Azure Platform is easy to use rather than Amazon (AWS).

\subsection{Organizational Category}

The organizational category refers to factors that involve organizational involvement or process. This category describes the manager's readiness in terms of finances to initiate and sustain the cloud usage, as well as resource readiness that consists of human and infrastructure to ensure knowledge development and stability $[4,35]$. The second category (organizational) consists of four factors; (i) infrastructure readiness, (ii) top management, (iii) knowledge and IT skillset and (iv) financial.

\subsubsection{Coding Unit 1: Infrastructure Readiness}

The infrastructure readiness consists of technological infrastructures such as hardware and software to support the usage of cloud computing. The other important element in infrastructure readiness is IT expertise that has knowledge and skill to implement cloud computing [36]. Knowledge and IT skillset is part of infrastructure readiness. The majority of the informants highlighted the importance of infrastructure readiness related to technological infrastructure.

\subsubsection{Coding Unit 2: Top Management}

The top management plays an important role in any department. They govern vision, mission, creating values, resources commitment to align with organization business goals [31]. The IT Centre has stated the department's direction towards cloud computing implementation in IT Strategic Planning 20152017. The top management support in terms of financial allocation, infrastructure readiness, knowledge development and training are important to align with the organization's aims. Hence, the support will ensure the resource readiness to adapt and innovate the technology.

\subsubsection{Coding Unit 3: Knowledge and IT skillset}

The learning curve in cloud computing is part of management readiness in ensuring the adoption of technology success [6]. The management should see the technology able to give the opportunity to develop and experience new skills that can contribute to a new paradigm of the working environment and job satisfaction [29]. 


\subsubsection{Coding Unit 4: Financial}

The financial become part of top management support towards technology innovation in the organization. The financial factor influenced the decision to operate IT services in the university in many ways. Today, the economic turbulent that give impact to many industries including the Malaysia government. The reduction budget from the government becomes a challenge to the higher education institutions to remain competitive [37]. Thus, the top management of the IT department very concerns about to use the budget allocation wisely. One approach from top management was moving some of the suitable applications towards cloud computing technology. Cloud computing offers pay per use billing model and lower cost initial setup helps the university to fulfil budget allocation. There were five quotations from two key informants' responses to this factor.

\subsection{Environmental Category}

The environmental category refers to the external factors that can influence the adoption of technologies [27]. The environmental category explains external element characteristics that may influence the adoption of cloud computing in a certain organization [1]. The environmental context is determined as the industry, competitors and governmental policy [36]. The third category (environmental) consists of five factors; (i) Cloud Service Provider, (ii) Geographical, (iii) Data Privacy, (iv) Guideline and Policy, (v) Service Level Agreement (SLA).

\subsubsection{Coding Unit 1: Cloud Service Provider}

The cloud service provider (CSP) is one of the stakeholders in a cloud computing environment. Undoubtedly, CSP supports the organization IT business such as collaborate with network players to ensure high availability architecture, storage technology partner to ensure fast and transparently unlimited usage to the customer, backup operation, development and test the platform [31].

\subsubsection{Coding Unit 2: Geographical}

The geographical can be described as a location where the data being stored and operated. This factor becomes important criteria in selecting the cloud service provider because the data stored may be influenced by legal and act as well as the political environment at the hosted country [33]. This factor also associated with the data privacy factor which concerns about the unauthorised party can use the data without permission from the customer. The university was concerned about the geographical location and has the intention to consider this factor as one of the criteria in choosing the cloud service provider.

\subsubsection{Coding Unit 3: Data Privacy}

The data privacy becomes a part of the security factor. The data on the cloud tends to be used purposely or not purposely by the cloud service provider. The customer may have less control and do not know who can access their data and leads to reluctant to host their data on the cloud [31]. This situation also concerns by the university. Hosted the university email on Google cloud platform leads to privacy issues by the U.S.A. government under the Patriot Act. There were two quotations with one key informant were highlight this factor.

\subsubsection{Coding Unit 4: Guideline and Policy}

The guidelines and policies are important to drive cloud computing adoption. The policy governed must compatible with the organization's needs. The policy is significant in ensuring customer business policies that concentrated on quality and security measures to ensure the integrity of their services [26]. There were two key informants with two quotations stressed about the guideline and policies in the interview sessions. As a government institution, the organization must apply to any relevant guidelines or policies.

\subsubsection{Coding Unit 5: Service Level Agreement (SLA)}

The SLA is an agreement between the client and the provider to minimize the risk of cloud computing service. SLA is important for the customer gaining confident, secure and safe when dealing with the cloud environment [32]. Unclear SLA will hinder from adopting the technology [26]. This factor has been linked to the cloud service provider factor because it involves customer and cloud service providers as the stakeholders in the public cloud environment. The factor has been stressed by the Acting IT Director during the interview session. SLA will ensure the service on cloud getting support as agreed between customer and cloud service provider. 


\section{CONCLUSION}

The cloud computing adoption issues in the university may be reduced by knowing and understanding the influencing factors during the adoption process. For example, proper planning and conducting cloud adoption analysis based on the identified factors would help the organization to choose suitable deployment and service models as well as the cloud provider. Besides, unsustainability operational cost and data privacy issues may also be reduced by understanding the resource's capability such as knowledge and IT skillset, budget and financial level by performing a cost-benefit analysis and developing internal cloud governance. The cloud governance can be initiated by creating guidelines and policies, negotiating and having clear cloud SLA, investigating legal and act, data geographical and level of data privacy impact. The cloud computing investigation based on the experience of the university will be more meaningful if future studies can measure the validity of these factors among other institutions of higher learning. The result of this study may provide a reference for the adoption of cloud computing in the area of mobile learning or mobile computing. Future work involves conducting similar studies at other case studies including public and private universities in Malaysia.

\section{ACKNOWLEDGEMENTS}

This research is funded by the Research Entity Initiative (REI), Universiti Teknologi MARA, Malaysia, (Project Code: 600-IRMI/REI 5/3 (013/2018)).

\section{REFERENCES}

[1] H. Sallehudin, R. C. Razak, and M. Ismail, "Factors Influencing Cloud Computing Adoption in the Public Sector: An Empirical Analysis," Journal of Entrepreneurship and Business, vol. 3, no. 1, pp. 30-45, 2015.

[2] L. G. Tornatzky and K. J. Klein, "Innovation characteristics and innovation adoption-implementation: A metaanalysis of findings," IEEE Transactions on Engineering Management, vol. EM-29, no. 1, pp. 28-45, 1982.

[3] E. M. Rogers, Diffusion of Innovations Theory (5th ed.), in New York, NY: Free Press, 2003.

[4] H. Gangwar, H. Date, and R. Ramaswamy, "Understanding determinants of cloud computing adoption using an integrated TAM-TOE model," Journal of Enterprise Information Management, vol. 28, no. 1, pp. 107-130, 2015.

[5] S. S. Alam and M. N. Ahsan, "ICT Adoption in Malaysian SMEs from Services Sectors : Preliminary Findings," Journal of Internet Banking and Commerce, vol. 12, no. 3, pp. 1-11, 2007.

[6] L. Morgan and K. Conboy, "Factor Affecting The Adoption Of Cloud Computing: An Exploratory Study," Proceedings of the 21st European Conference on Information Systems, pp. 1-12, 2013.

[7] P. Mell and T. Grance, "The NIST Definition of Cloud Computing Recommendations of the National Institute of Standards and Technology," National Institute of Standards and Technology, vol. 145, p. 7, 2011.

[8] D. G. Chandra, "Role of Cloud Computing in Education," 2012 International Conference on Computing, Electronics and Electrical Technologies (ICCEET), pp. 832-836, 2012.

[9] R. Buyya, J. Broberg, and A. Goscinski, Cloud Computing: Principles and Paradigms, Wiley, 2011.

[10] N. Alsaeed and M. Saleh, "Towards Cloud Computing Services for Higher Educational Institutions: Concepts \& Literature Review," 2015 International Conference on Cloud Computing (ICCC), pp. 1-7, 2015.

[11] S. Okai, M. Uddin, A. Arshad, R. Alsaqour, and A. Shah, "Cloud Computing Adoption Model for Universities to Increase ICT Proficiency," SAGE Open, vol. 4, no. 3, pp 1-10, 2014.

[12] D. Dang-Pham and M. Z. Nkhoma, "Contributing Factors of Cloud Computing Adoption: a Technology Organisation - Environment Framework Approach," International Journal of Information Systems and Engineering, vol. 1, no. 1, pp. 38-49, 2013.

[13] R. Baniwal, “Applications of Cloud Computing in Different Areas,” IJCSC, vol. 4, no. 2, pp. 174-176, 2013.

[14] N. Gupta and S. Thakur, "The Factors Affecting Adoption of Cloud Computing Technology in Education Institutions," International Journal of Advances Research in Computer and Communication Engineering, vol. 3, no. 6, pp. 7229-7235, 2014.

[15] M. Anshari, Y. Alas, and L. S. Guan, "Developing online learning resources: Big data, social networks, and cloud computing to support pervasive knowledge," Education and Information Technologies, vol. 21, no. 6, pp. 1663$1677,2016$.

[16] P. Ganesh, K. Sailaja Kumar, D. Evangelin Geetha, T V Suresh Kumar, "Performance evaluation of cloud service with hadoop for twitter data," Indonesian Journal of Electrical Engineering and Computer Science (IJEECS), vol. 13, no. 1, pp. 392-404, 2019.

[17] R. Wishah, M. Al-Nsour, M. Alharafsheh, S. AlKhalaileh, F. Alshbeekat, "Factors influencing cloud computing in telecommunications companies," Indonesian Journal of Electrical Engineering and Computer Science (IJEECS),, vol. 12, no. 3, pp. 1334-1339, 2018.

[18] W. A. R. Wan Mohd Isa, A. I. H. Suhaimi, N. Noordin, A. F. Harun, J. Ismail, R. Awang Teh, "Cloud computing adoption reference model," Indonesian Journal of Electrical Engineering and Computer Science (IJEECS),, vol. 16, no. 1 , pp. $395-400,2019$. 
[19] S. A. Mokhtar, S. H. Shaikh Ali, A. Al-Othman, and A. Al-Sharafi, "Identifying the Determinants of Cloud Computing Adoption in Higher Education Institutions," 2016 International Conference on Information Communication Technology (ICICTM), 2016.

[20] R. F. El-Gazzar, "Cloud computing adoption factors and processes for enterprises," Proceedings of the 4th International Conference on Cloud Computing Services Science (CLOSER 2014), pp. 78-87, 2014.

[21] J. W. Creswell, Educational Research: Planning, Conducting, and Evaluating Quantitative and Qualitative Research, Pearson, 2012.

[22] M. Hennink, Inge Hutter, and A. Bailey, "Qualitative research methods," Critical Public Health, vol. 22, no. 1. pp. $111-112,2012$.

[23] J. Mason, Qualitative Researching, SAGE Publishing, 2013.

[24] R. K. Yin, Case study research: Design and methods, SAGE, 2014.

[25] S. U. Mohd Tobi, Qualitative Research, Interview Analysis \& Nvivo 11 Exploration, ARAS, 2016.

[26] A. Lin and N. C. Chen, "Cloud computing as an innovation: Perception, attitude, and adoption," International Journal of Information Management, vol. 32, no. 6, pp. 533-540, 2012.

[27] A. Saedi and N. A. Iahad, "An Integrated Theoretical Framework for Cloud Computing Adoption by Small and Medium-Sized Enterprises,” Pacific Asia Conference on Information Systems (PACIS), 2013.

[28] P. K. Ross and M. Blumenstein, "Cloud computing as a facilitator of SME entrepreneurship," Technology Analysis \& Strategic Management, vol. 27, no. 1, pp. 87-101, 2015.

[29] T. Kihara and D. Gichoya, "Adoption and Use of Cloud Computing in Small and Medium Enterprises in Kenya," 2013 IST-Africa Conference \& Exhibition, 2013.

[30] F. Davis, "Perceived Usefulness, Perceived Ease of Use, and User Acceptance of Information Technology," MIS Quarterly, vol. 13, no. 3, pp. 319-340, 1989.

[31] H. Gangwar, H. Date, and R. Ramaswamy, "Understanding determinants of cloud computing adoption using an integrated TAM-TOE model," Journal of Enterprise Information Management, vol. 28, no. 1, pp. 107-130, 2015.

[32] M. Stieninger, D. Nedbal, W. Wetzlinger, G. Wagner, and M. A. Erskine, "Impacts on the organizational adoption of cloud computing: A reconceptualization of influencing factors," Procedia Technology, vol. 16, pp. 85-93, 2014.

[33] M. Stieninger and D. Nedbal, "Diffusion and Acceptance of Cloud Computing in SMEs : Towards a Valence Model of Relevant Factors," 2014 47th Hawaii International Conference System Sciences, 2014.

[34] V. Chang, G. Wills, and D. De Roure, "A Review of Cloud Business Models and Sustainability," 2010 IEEE 3 rd International Conference on Cloud Computing, 2010.

[35] H. S. Hashim, Z. B. Hassan, and A. S., Hashim, "Factors Influence the Adoption of Cloud Computing: A Comprehensive Review," International Journal of Education and Research, vol. 3, no. 7, pp. 295-306, 2015.

[36] C. Low, Y. Chen, and M. Wu, "Understanding the determinants of cloud computing adoption," Industrial Management \& Data Systems, vol. 111, no. 7, pp. 1006-1023, 2011.

[37] K. A. Ratnam, P. D. D. Dominic, and T. Ramayah, "A structural equation modeling approach for the adoption of cloud computing to enhance the Malaysian healthcare sector systems-level quality improvement," Journal of Medical Systems, vol. 38, no. 8, 2014. 